\title{
Erziehungsbezogene elterliche Depressivität und Selbstwirksamkeitserwartung von Kindern: Die Rolle von Freundschaftsqualität
}

\author{
Olivia Gasser-Haas ${ }^{1}$, Fabio Sticca ${ }^{1}$, Corina Wustmann Seiler ${ }^{2}$
}

Marie Meierhofer Institut für das Kind, Zürich, Schweiz

2 Pädagogische Hochschule Zürich, Schweiz

Zusammenfassung: Die Studie untersuchte die Auswirkungen erziehungsbezogener elterlicher Depressivität auf die Selbstwirksamkeitserwartung bei 189 Kindern im Alter zwischen 9-11 Jahren $\left(M_{\text {Alter }}=9.69, S D_{\text {Alter }}=0.48\right)$. Dabei interessierte die promotive und protektive Rolle der Freundschaftsqualität zum besten Freund beziehungsweise zur besten Freundin.

Die erziehungsbezogene elterliche Depressivität wurde bei den Eltern über das Eltern-BelastungsInventar (EBI) von Tröster (2011) erfasst. Ebenso schätzten die Eltern die (allgemeine) Selbstwirksamkeitserwartung ihres Kindes mithilfe der Skala von Jerusalem und Schwarzer (1999) ein. Die Kinder beurteilten ihre Freundschaftsqualität zum besten Freund beziehungsweise zur besten Freundin anhand des Friendship Quality Questionnaire von Parker und Asher (1993).

Die Ergebnisse zeigten, dass eine höhere erziehungsbezogene elterliche Depressivität mit weniger Selbstwirksamkeitserwartung der Kinder einhergeht. Die Freundschaftsqualität zum besten Freund beziehungsweise zur besten Freundin erweist sich in diesem Zusammenhang sowohl als promotiv als auch als protektiv für die kindliche Selbstwirksamkeitserwartung. Die Bedeutsamkeit bester Freundschaften im Schulalter werden diskutiert und Schlussfolgerungen ausgeführt.

Schlüsselbegriffe: Erziehungsbezogene elterliche Depressivität, Selbstwirksamkeitserwartung, Freundschaftsqualität, Schutzwirkung

\section{Parenting-related Depressiveness and Children's Self-Efficacy: The Role of Friendship Quality}

Summary: The study examined the effect of parenting-related depressiveness on the general selfefficacy of 189 children aged $9-11$ years $\left(M_{\text {age }}=9.69, S D_{\text {age }}=0.48\right)$. Of particular interest was the promotive and protective role of children's friendship quality to their best friend. Parenting-related depressiveness was assessed with the Parental Stress Index (ECI) by Tröster (2011). The parents also assessed their child's (general) self-efficacy using the scale of Jerusalem and Schwarzer (1999). The children self-perceived the friendship quality to their best friend based on the Friendship Quality Questionnaire by Parker and Asher (1993).

The results showed that higher parenting-related depressiveness was associated with lower child self-efficacy.

Quality of friendship to the best friend was found to be both promotive and protective for children's self-efficacy. The importance of best friendships at school age will be discussed and conclusions suggested.

Keywords: Parental depressiveness, Self-efficacy, Friendship quality, Protective effect 


\section{Erziehungsbezogene elterliche Depressivität und Selbstwirksamkeits- erwartung von Kindern: Die Rolle von Freundschaftsqualität}

Die allgemeine Selbstwirksamkeitserwartung wird als die subjektive Überzeugung der generellen Lebensbewältigungskompetenz beschrieben (Schwarzer \& Jerusalem, 2002). Nach der sozial-kognitiven Theorie von Bandura (1997) gilt die Selbstwirksamkeitserwartung als ein Schlüsselfaktor für das menschliche Handeln. Verschiedene Studien weisen darauf hin, dass die Selbstwirksamkeitserwartung für das Wohlbefinden, die psychische Gesundheit und für akademische Leistungen zentral ist (Bandura, Pastorelli, Barbaranelli \& Caprara, 1999; Caprara, Barbaranelli, Pastorelli \& Cervone, 2004; Ebner, Schulte, Soucek \& Kauffeld, 2018; Graber, Turner \& Madill, 2016; McCauley, Weymouth, Feinberg \& Fosco, 2019).

Fehlende elterliche Unterstützung, mangelndes elterliches Monitoring sowie eine geringe Responsivität beeinträchtigen die Selbstwirksamkeitserwartung in der frühen und mittleren Kindheit maßgebend (Hohm et al., 2017). Eine der bekanntesten Belastungs- und Stressquellen in der Erziehung stellt die erziehungsbezogene elterliche Depressivität dar (Abidin, 1995; Belsky, 1984; Östberg, 1998). Es kann vermutet werden, dass eine erziehungsbezogene elterliche Depressivität sich ebenso als Risikofaktor für die Entwicklung kindlicher Selbstwirksamkeitserwartung zeigt. Unter erziehungsbezogener elterlicher Depressivität wird eine eingeschränkte emotionale Verfügbarkeit von Eltern in der Erziehung ihres Kindes verstanden, infolge depressiver Kognitionen und Emotionen (Tröster, 2011).

Während die einen Kinder psychische Beeinträchtigungen bei einer hohen Risikobelastung aufweisen, entwickeln sich andere Kinder trotz hoher Belastung positiv (Dutra-Thomé, DeSousa \& Koller, 2018; Laucht, Esser \& Schmidt, 2000; Masten, Best \& Garmezy, 1990; Wustmann Seiler, 2020). Grund dafür sind schützende, risikomildernde Faktoren, die nach Rutter (1990) die Auftretenswahrscheinlichkeit psychischer Störungen senken oder die Auftretenswahr- scheinlichkeit einer positiven Entwicklung erhöhen. Ein Schutzfaktor kann dabei gleichzeitig promotiv (entwicklungsförderlich) und protektiv (schützend) sein oder sich nur als promotiv beziehungsweise nur als protektiv erweisen (Burke, Sticca \& Perren, 2017; Scheithauer \& Petermann, 1999; Masten \& Barnes, 2018; Rutter, 1990; Wustmann Seiler, Müller \& Simoni, 2017). Untersuchungen auf der Basis der interpersonalen Theorie von Sullivan (1953) belegen, dass eine hohe Freundschaftsqualität zum besten Freund beziehungsweise zur besten Freundin ein promotives und protektives soziales Unterstützungssystem bei vorhandenen elterlichen Belastungen sein kann (Lansford, Criss, Pettit, Dodge \& Bates, 2003).

Stützend auf die Theorien von Bandura (1997) und Sullivan (1953) wurde in der vorliegenden Studie die Bedeutung erziehungsbezogener elterlicher Depressivität für die kindliche Selbstwirksamkeitserwartung untersucht und dabei die Rolle der Freundschaftsqualität zum besten Freund beziehungsweise zur besten Freundin als promotiver und/oder protektiver Faktor beleuchtet.

\section{ZurRolle erziehungsbezogener elterlicher Depressivität für die Entwicklung kind- licher Selbstwirksamkeitserwartung}

Bandura (1997) geht von vier Hauptquellen für den Erwerb der Selbstwirksamkeitserwartung aus, die nach der Stärke ihres Einflusses geordnet sind: (1) direkt erlebte Handlungserfolge und Misserfolge, (2) stellvertretende Erfahrungen durch Beobachtung von Verhaltensmodellen, (3) verbal ausgedrückte Überzeugungen und Ermutigungen (z. B. Fremdbewertung oder Selbstinstruktion) sowie (4) Wahrnehmungen eigener Gefühlserregung. Alle vier Quellen, dabei insbesondere die Fremdbewertung und das Erleben eigener Erfolge und Misserfolge, sind eng an die Erfahrungen im familiären Nahumfeld geknüpft (Hohm et al., 2017). Das erzieherische Handeln der Eltern sowie die Interaktionen zwischen Eltern und Kind können als wesentliche Einflussfaktoren der Selbstwirksamkeitserwartung beschrieben werden (Bandura, 1997; Hohm et al., 2017). 
Das Erziehungsverhalten von Eltern mit einer erziehungsbezogenen Depressivität unterscheidet sich von demjenigen bei Eltern ohne psychische Belastung in der Erziehung (Hammen, 1997; Kötter, Stemmler, Bühler \& Lösel, 2010). Verschiedene Studien belegen, dass die Qualität der Eltern-Kind-Beziehung bei vorhandener erziehungsbezogener elterlicher Depressivität massiv beeinträchtigt sein kann (Lenz, 2008; World Health Organization, 2004). Nach Lenz und Kuhn (2011) zeigen betroffene Eltern ihren Kindern gegenüber weniger Interesse, emotionale Beteiligung und Feinfühligkeit. Vermehrte Passivität und negative Gefühle, wie Selbstvorwürfe und Selbstzweifel in der Erziehung, sind weitere Merkmale von Eltern mit erziehungsbezogener Depressivität (Lenz, 2008, 2014; Tröster, 2011; Venkataraman \& Ackerson, 2008).

Die erziehungsbezogene elterliche Depressivität, ähnlich der diagnostizierten, generellen Depression, wurde bereits mehrfach als Risikofaktor für die Gesundheit und die sozial-emotionale Entwicklung von Kindern identifiziert (Cummings \& Davies, 1994; Goodman, 2007; Hammen, 1997; Kötter et al., 2010; Lenz \& Kuhn, 2011; Tröster, 2011). So haben betroffene Kinder eine höhere Wahrscheinlichkeit, selbst eine psychische Störung zu entwickeln (Lenz \& Kuhn, 2011), und neigen öfter zu internalisierenden sowie externalisierenden Problemen (Bloomfield \& Kendall, 2012; Cummings \& Davies, 1994; Gelfand \& Teti, 1990; Hammen, 1997). Auch wenn eine erziehungsbezogene elterliche Depressivität als Teilaspekt einer diagnostizierten, generellen Depression verstanden werden kann, handelt es sich hierbei um zwei unterschiedliche Konzepte. Während für eine Depression eine Diagnosestellung im Sinne einer klinischen, kategorialen Diagnose erforderlich ist (ICD-10), kann eine erziehungsbezogene elterliche Depressivität unabhängig von einer diagnostizierten Depression vorhanden sein und unterschiedliche Merkmalsausprägungen von niedrig bis hoch aufweisen (Kotov et al., 2017; Schmitt \& Altstötter-Gleich, 2010).
Untersuchungen zur mütterlichen Depression zeigen, dass Kinder im Alter von 18 Monaten eine deutlich niedrigere Selbstwirksamkeitserwartung aufweisen als Kinder von Müttern ohne Depression (Jennings \& Abrew, 2004). Darüber hinaus wiesen verschiedene Forschende auf die intergenerationale Transmission einer Depression hin, bei welcher auf eine verminderte Selbstwirksamkeitserwartung von Kindern bei mütterlicher Depression verwiesen wurde (Cummings \& Davies, 1994; Jennings \& Abrew, 2004).

Zum Einfluss der erziehungsbezogenen Depressivität von Eltern auf die Entwicklung der kindlichen Selbstwirksamkeitserwartung liegen noch keine Befunde vor. Es kann jedoch davon ausgegangen werden, dass eine vorhandene erziehungsbezogene elterliche Depressivität einen negativen Einfluss auf die Selbstwirksamkeitserwartung von Kindern hat.

\section{Zur Rolle der Freundschaftsqualität als promotiver und protektiver Faktor}

Beste Freundschaften gewinnen im Alter von neun bis elf Jahren entwicklungsbedingt an Bedeutung; sie werden intimer und einflussreicher (Bagwell \& Bukowski, 2018; Hartup, 1989; Havighurst, 1976; Rubin \& Bowker, 2018; Sullivan, 1953). Folglich können beste Freunde beziehungsweise beste Freundinnen, nebst den eigenen Familienmitgliedern, zu den wichtigsten Bezugspersonen für Kinder im mittleren Schulalter gezählt werden (Waldrip, Malcolm \& Jensen-Campbell, 2008). Die Freundschaftsqualität zum besten Freund beziehungsweise zur besten Freundin zeichnet sich durch positive Qualitätsdimensionen wie Verständnis, Nähe, Verbundenheit und wechselseitige Anerkennung aus und entwickelt sich maßgeblich im Alter von neun bis elf Jahren (Berndt, 2002; Parker \& Asher, 1993; Sullivan, 1953; Traub, 2006). Eine hohe Freundschaftsqualität führt im Sinne eines promotiven Unterstützungssystems zu mehr Glück, Selbstwertgefühl und Wohlbefinden (Bagwell \& Bukowski, 2018; Bakalım \& Karçkay, 2016; Demir, Özdemir \& Weitekamp, 
2007; Hoza, Bukowski \& Beery, 2000; Rubin \& Bowker, 2018). Beste Freunde beziehungsweise beste Freundinnen hoher Qualität können einander emotional unterstützen und Ratschläge im Umgang mit schwierigen Situationen geben (Bagwell \& Schmidt, 2011; Bukowski, Laursen \& Rubin, 2018; Nangle, Erdley, Newman, Mason \& Carpenter 2003; Oldenburg \& Kems, 1997; Parker \& Asher, 1993; Sanderson \& Siegal, 1995; Schmidt \& Bagwell, 2007; Schneider, 2016). Des Weiteren werden sie als Unterstützungspersonen in der Weiterentwicklung von sozial-emotionalen Kompetenzen verstanden (Bagwell \& Bukowski, 2018; Bagwell \& Schmidt, 2011; Rodebaugh, Lim, Shumaker, Levinson \& Thompson, 2015; Simoni, Herren, Kappeler \& Licht, 2015).

Studien weisen darauf hin, dass eine hohe Freundschaftsqualität negative Einflüsse familialer und peerbezogener Belastungen auf die sozial-emotionale Entwicklung von Kindern und Jugendlichen im Sinne eines protektiven Unterstützungssystems abzupuffern vermag (Bagwell \& Schmidt, 2011; Bukowski et al., 2018; Gaertner, Fite \& Colder, 2010; Lansford et al., 2003; Nangle et al., 2003; Parker \& Asher, 1993; Sanderson \& Siegal, 1995; Schmidt \& Bagwell, 2007). So konnten Cuadros und Berger (2016) zeigen, dass die negativen Auswirkungen von Ablehnung und Mobbing auf Einsamkeit und Depression durch eine hohe Freundschaftsqualität abgeschwächt werden können. Auch kann der negative Einfluss dysfunktionaler Erziehung, wie einseitige elterliche Entscheidungsfindung, geringes Monitoring sowie inkonsistente und harte Disziplin in der Erziehung, auf internalisierende und externalisierende Probleme von Kindern durch eine hohe Freundschaftsqualität gemildert werden (Gaertner et al., 2010; Lansford et al., 2003). Furman und Buhrmester (1985) betonen ebenfalls die Kompensationsfunktion bester Freundschaften bei elterlicher Dysfunktionalität. Basierend auf den vorliegenden Forschungsergebnissen kann davon ausgegangen werden, dass sich die Freundschaftsqualität zum besten Freund beziehungsweise zur besten Freundin im Zusammenhang von erzie- hungsbezogener elterlicher Depressivität und kindlicher Selbstwirksamkeitserwartung sowohl als promotiv als auch als protektiv erweist.

\section{Fragestellungen}

Die folgenden Fragestellungen standen im Zentrum der Untersuchung: (1) Inwiefern hängt die erziehungsbezogene Depressivität von Eltern mit der Selbstwirksamkeitserwartung von neunbis elfjährigen Kindern zusammen? (2) Welche Rolle spielt die Freundschaftsqualität zum besten Freund beziehungsweise zur besten Freundin als promotiver und/oder protektiver Faktor im Zusammenhang erziehungsbezogener elterlicher Depressivität und kindlicher Selbstwirksamkeitserwartung? Dabei wurde davon ausgegangen, dass die erziehungsbezogene elterliche Depressivität negativ mit der kindlichen Selbstwirksamkeitserwartung zusammenhängt ( $\mathrm{H1})$ und die Freundschaftsqualität als promotiver Faktor einen positiven Effekt auf die kindliche Selbstwirksamkeitserwartung zeigt (H2). Des Weiteren wurde angenommen, dass eine hohe Freundschaftsqualität zum besten Freund beziehungsweise zur besten Freundin den $\mathrm{Zu}$ sammenhang zwischen erziehungsbezogener elterlicher Depressivität und kindlicher Selbstwirksamkeitserwartung abschwächt und als Schutzfaktor fungiert (H3).

\section{Methode}

\section{Stichprobe}

Die Studie wurde in der Schweiz im Rahmen der Längsschnittstudie „Langfristige Auswirkungen früher familialer Risiken auf Verhaltensprobleme und die Selbstwirksamkeit - Individuelle, familiale und außerfamiliale Schutzprozesse" (2016-2019) durchgeführt. Die Stichprobe bestand aus 189 Kindern (48,6\% weiblich) im Alter von neun bis elf Jahren $\left(M_{\text {Alter }}=9.69, S D_{\text {Alter }}=0.48\right)$ und deren Eltern. Bereits sieben Jahre zuvor, im Jahr 2009, wurden die Kinder und Eltern aus 25 Kindertagesstätten für die Ursprungsstudie „Bildungs- und Resilienzförderung im Frühbereich" (2009-2012) (Wustmann Seiler et al., 2017) rekrutiert $\left(N=293,47,9 \%\right.$ weiblich, $M_{\text {Alter }}=2.81$, $\left.S D_{\text {Alter }}=0.55\right)$. Die aktuelle Studie bezieht sich aus- 
schließlich auf den letzten Messzeitpunkt, da erziehungsbezogene Stress- und Belastungsquellen sowie die Freundschaftsqualität zum besten Freund beziehungsweise zur besten Freundin erstmals zu diesem Zeitpunkt erfasst wurden. Von den insgesamt $189 \mathrm{Fa}-$ milien wiesen rund $70 \%$ der Mütter und $74 \%$ der Väter zum Zeitpunkt der Fortsetzungsstudie einen Universitätsabschluss vor. Die Kinder sprachen zu Hause hauptsächlich Deutsch, lediglich 12\% der Kinder hatten einen fremdsprachigen Hintergrund.

Rund $80 \%$ der Kinder lebten gemeinsam mit ihren Eltern in einem Haushalt, 10\% lebten mit einem Elternteil und weitere $10 \%$ mit einem Elternteil und deren neuer Partnerschaft im Haushalt.

\section{Datenerhebung}

Eltern und Kinder wurden mündlich und schriftlich über das Vorgehen, die Ziele und den Ablauf der Datenerhebung informiert. Beide gaben eine schriftliche Einverständniserklärung für die Teilnahme an der Studie ab. Jederzeit und ohne Angabe von Gründen war ein Abbruch der Studienteilnahme möglich. Geschulte Mitarbeiterinnen besuchten die Familien zu Hause und führten die Interviews mit den Eltern und Kindern durch. Während die Eltern den Großteil der Fragen in einem strukturierten Interview beantworteten, wurden sensible Fragen in einem ergänzenden Fragebogen ausgefüllt. Den Fragebogen für die Kinder ging die Mitarbeiterin gemeinsam mit dem Kind durch, um sicherzugehen, dass alle Fragen richtig verstanden werden. Die Kinder erhielten zum Abschluss einen Büchergutschein als Dank für die Teilnahme. Sämtliche Daten wurden nach Beendigung der Datenerhebung anonymisiert.

\section{Erhebungsinstrumente}

\section{Erziehungsbezogene elterliche Depressivität}

Zur Erfassung der erziehungsbezogenen Depressivität der Eltern wurde die Skala Depression des Eltern-Belastungs-Inventars (EBI) von Tröster (2011) eingesetzt. Das EBI besteht aus 48 Items und ist die deutsche Version der dritten Auflage des Parenting Stress Index (PSI) von Abidin (1995). In zahlreichen Studien wurde das EBI erfolgreich als ScreeningVerfahren eingesetzt, um elterliche Belastungen in der Erziehung, Betreuung und Versorgung der Kinder zu erfassen (z. B. Bloomfield \& Kendall, 2012; Östberg, 1998; Schaunig et al., 2004). Die Skala
Depression erfragt Schuldgefühle und Selbstvorwür$\mathrm{fe}$ in der Erziehung und umfasst vier Items, welche anhand einer 4-Punkte-Likert-Skala von 1 (Trifft nicht zu) bis 4 (Trifft genau zu) von einem Elternteil eingeschätzt wurden: „Ich habe manchmal das Gefühl, es ist eigentlich meine Schuld, wenn mein Kind etwas verkehrt gemacht hat", „Ich denke manchmal, dass ich etwas falsch mache, wenn mein Kind sich schlecht benimmt oder zu viel quengelt“, „Es bedrückt mich, wenn ich merke, dass ich gereizt auf mein Kind reagiere“ und „Es bedrückt mich, wenn ich darüber nachdenke, was man in der Erziehung alles falsch machen kann“. Die Reliabilität (McDonald's Omega) lag bei $\omega=.71$.

\section{Kindliche Selbstwirksamkeitserwartung}

Anhand der Skala zur allgemeinen Selbstwirksamkeitserwartung von Jerusalem und Schwarzer (1999) schätzten die Eltern die Selbstwirksamkeitserwartung ihres Kindes ein. Die Skala besteht aus insgesamt zehn Items, die anhand einer 4-Punkte-LikertSkala von 1 (Trifft überhaupt nicht zu) bis 4 (Trifft voll und ganz $\mathrm{zu}$ ) reichen. Eine konfirmatorische Faktorenanalyse zeigte, dass die Varianzaufklärung von fünf Items durch die latente Variable weniger als $25 \%$ betrug, weshalb diese nach Hulland (1999) und Hair (2014) ausgeschlossen wurden. Die folgenden fünf Items wurden in das Modell aufgenommen: „In unerwarteten Situationen weiß mein Kind immer, wie es sich verhalten soll“, „Schwierigkeiten sieht mein Kind gelassen entgegen, weil es den eigenen Fähigkeiten immer vertrauen kann“, „Mein Kind weiß: Was auch immer passiert, es wird schon klarkommen“, „Für jedes Problem kann mein Kind eine Lösung finden“ und „Wenn eine neue Sache auf mein Kind zukommt, weiß es, wie es damit umgehen kann". McDonald's Omega lag bei $\omega=.82$.

\section{Freundschaftsqualität zum besten Freund beziehungsweise zur besten Freundin}

Die Freundschaftsqualität zum besten Freund beziehungsweise zur besten Freundin wurde mit dem Friendship Quality Questionnaire (FQQ; Parker \& Asher, 1993) erhoben. Die Skala besteht aus 40 Items mit fünf positiven Komponenten (Hilfe und Beratung, Intimer Austausch, Bestätigung und Fürsorge, Gemeinschaft und Freizeit sowie Konfliktlösung) und einer negativen Komponente (Konflikt und Verrat). Das Antwortformat beinhaltete eine 5-PunkteLikert-Skala von 0 (Überhaupt nicht wahr) bis 4 
(Absolut wahr). Vor dem Ausfüllen des Fragebogens wurden die Kinder gebeten, den Namen ihres besten Freundes beziehungsweise ihrer besten Freundin aufzuschreiben, um einen eindeutigen Bezug herzustellen. In die vorliegende Studie gingen die Items von vier positiven Komponenten ein: Hilfe und Beratung (z. B. „[Beste/r Freund/in] und ich tun uns gegenseitig besondere Gefallen"), Intimer Austausch (z. B. „, [Beste/r Freund/in] und ich sprechen über die Dinge, die uns traurig machen"), Bestätigung und Fürsorge (z. B. „[Beste/r Freund/in] gibt mir ein gutes Gefühl, wenn es um meine Ideen geht") und Konfliktlösung (z. B. „,[Beste/r Freund/in] und ich überwinden unsere Streitigkeiten immer ziemlich schnell“). Die positive Komponente Gemeinschaft und Freizeit wurde nicht in die Analyse einbezogen, da die Items einen gemeinsamen Schulbesuch der Freunde erfordern (z. B. ,[Beste/r Freund/in] und ich spielen in den Pausen immer gemeinsam"), was in der vorliegenden Studie nicht bei allen Kindern der Fall war. Des Weiteren wurde die negative Komponente der Freundschaftsqualität (Konflikt und Verrat) nicht berücksichtigt, da diese als negative Dimension der Freundschaftsqualität umschrieben wird und als potenzieller promotiver und protektiver Faktor laut Bagwell und Schmidt (2011) und Bukowski et al. (2018) auszuschließen ist. Von den vier in die Analyse einbezogenen Komponenten betrug McDonald's Omega $\omega=.74-.88$ (Hilfe und Beratung, $\omega=.85$; Intimer Austausch, $\omega=.88$; Bestätigung und Fürsorge, $\omega=.87$; Konfliktlösung, $\omega=.74$ ).

Für die Analyse wurden in einem ersten Schritt Mittelwerte der vier Komponenten gebildet, um diese in einem zweiten Schritt als manifeste Indikatoren der latenten Freundschaftsqualität zu modellieren (McDonald's Omega $\omega=.91$ ). Damit war es möglich, die Komplexität des Interaktionsterms zur Testung des Moderationseffekts zu reduzieren.

\section{Analysestrategie}

Zur Beantwortung der Fragestellungen wurde ein Strukturgleichungsmodell mit Mplus Version 8.0 (Muthén \& Muthén, 1998-2017) berechnet. Dabei wurde der robuste Maximum-Likelihood-Schätzer (MLR) verwendet und das Full-Information-Maximum-Likelihood-Verfahren (FIML) für fehlende Daten angewandt. Die Analyse des Modells erfolgte in zwei Schritten, wobei alle Konstrukte latent modelliert wurden. Als Erstes wurde der Haupteffekt der erziehungsbezogenen elterlichen Depressivität auf die
Selbstwirksamkeitserwartung der Kinder modelliert, während in einem zweiten Schritt die Freundschaftsqualität als Moderator in die Analyse einging. Dabei wurde der promotive Effekt über den Haupteffekt der Freundschaftsqualität auf die Selbstwirksamkeitserwartung der Kinder und der protektive Effekt über den Interaktionsterm der Freundschaftsqualität mit der erziehungsbezogenen elterlichen Depressivität auf die Selbstwirksamkeitserwartung der Kinder modelliert.

Der Moderationseffekt wurde mit der latenten Orthogonalisierungsmethode getestet (Little, 2013), die es ermöglicht, den Interaktionsterm zur Berechnung des Moderationseffekts von zwei latenten Variablen ebenfalls latent zu modellieren. Hierzu werden alle möglichen Interaktionsterme zwischen den manifesten Indikatoren mit den entsprechenden latenten Variablen multipliziert, hier also $4 \times 4=16$ neue Items gebildet. Jedes einzelne dieser multiplizierten Items wird dann auf alle Items regrediert, hier also 16 Items. Die Residuen dieser Regressionsmodelle werden als 16 neue Variablen abgespeichert, die dann als Indikatoren der latenten Interaktionsvariable fungieren können. Dadurch ergibt sich eine latente Interaktionsvariable, die mit beiden Ursprungsvariablen zu null korreliert ist („orthogonal“). Die Vorteile dieser Methode liegen darin, dass (1) der Interaktionsterm messfehlerfrei ist, (2) Multikollinearität vermieden wird, (3) die unbedingten Haupteffekte nicht verändert werden (also nicht zu bedingten Haupteffekten mutieren) und somit die Interpretation erleichtert wird, sowie (4) eine standardisierte Lösung in Mplus ausgegeben werden kann, was bei der XWITH Funktion (,latent random effect") nicht der Fall ist.

Geschlecht und Alter der Kinder wurden als Kovariaten ins Modell aufgenommen; sie wurden als manifeste Prädiktoren der Selbstwirksamkeitserwartung modelliert sowie mit den restlichen Variablen korreliert. Das Modell passte sehr gut auf die Daten $\left[\chi^{2}(374)=231,45 ; p<.001 ; \mathrm{CFI}=1.00 ; \mathrm{RMSEA}=.00\right.$; $\mathrm{SRMR}=.05]$ (Hu \& Bentler, 1999; Kline, 2016).

\section{Ergebnisse}

Im Folgenden sind die Ergebnisse der Analysen dargelegt. Tabelle 1 zeigt die Interkorrelationen zwischen allen Untersuchungsvariablen sowie deren Mittelwerte und Standardabweichungen. Die standardisierten Koeffizienten des Strukturgleichungsmodells sind in Abbildung 1 dargestellt. 
Tab. 1: Mittelwerte, Standardabweichungen und Interkorrelationen der Untersuchungsvariablen $(N=189)$

\begin{tabular}{|c|c|c|c|c|c|c|}
\hline Variable & $M$ & $S D$ & 1 & 2 & 3 & 4 \\
\hline 1 Erziehungsbezogene elterliche Depressivität & 2.30 & 0.54 & - & & & \\
\hline 2 Kindliche Selbstwirksamkeitserwartung & 3.00 & 0.39 & $-.33^{* * *}$ & - & & \\
\hline 3 Freundschaftsqualität & 2.96 & 0.70 & .00 & $.24^{*}$ & - & \\
\hline 4 Geschlecht (männlich) & 0.49 & - & .03 & -.17 & $-.23^{*}$ & - \\
\hline 5 Alter (Jahre) & 9.68 & 0.47 & -.12 & -.09 & -.09 & -.01 \\
\hline
\end{tabular}

Anmerkungen: Variablen $1-3$ sind latent modelliert. ${ }^{*} p<.05 ;{ }^{* *} p<.01 ;{ }^{* * *} p<.001$.

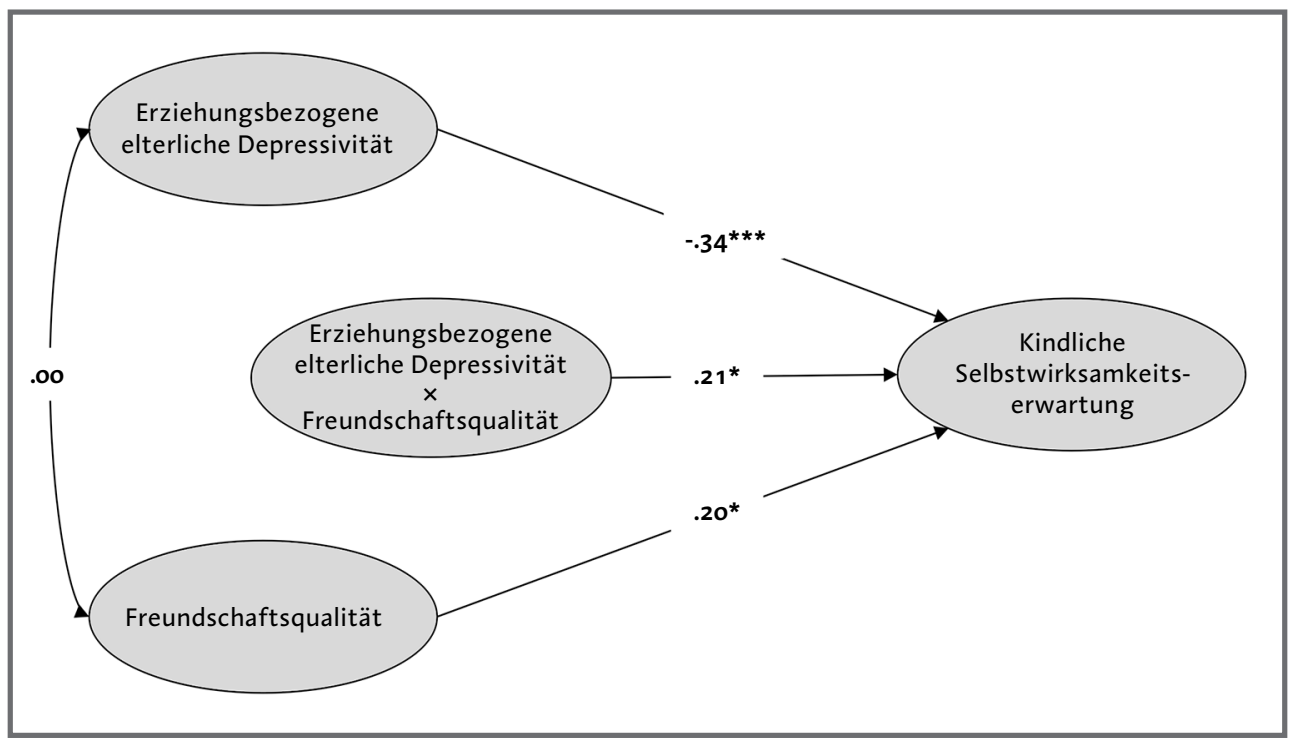

Abb. 1: Standardisierte Koeffizienten zur erziehungsbezogenen elterlichen Depressivität als unabhängige Variable und der Freundschaftsqualität als Moderator (Interaktion Erziehungsbezogene elterliche Depressivität $\times$ Freundschaftsqualität) zwischen erziehungsbezogener elterlicher Depressivität und kindlicher Selbstwirksamkeitserwartung. Modellgüte: $\left[\chi^{2}(374)=231.45, p<.001\right.$; CFI = 1.00; RMSEA =.00; SRMR =.05]. ${ }^{*} p<.05 ;{ }^{* * *} p<.001$.

\section{Haupteffekt ( $\left.\mathrm{H}_{1}\right)$}

In den Analysen zeigte sich, dass gemäß Hypothese (H1) die erziehungsbezogene elterliche Depressivität negativ mit der kindlichen Selbstwirksamkeitserwartung zusammenhängt. Der Effekt war negativ, mittelgroß und signifikant $(\beta=-.34, p<.01)$.

\section{Promotiver Effekt der Freundschaftsqualität ( $\left.\mathrm{H}_{2}\right)$}

Die Freundschaftsqualität zum besten Freund beziehungsweise zur besten Freundin zeigte einen signifikanten, kleinen bis mittelgroßen promotiven Effekt auf die kindliche Selbstwirksamkeitserwartung $(\beta=.20, p<.05)$.

\section{Protektiver Effekt der Freundschaftsqualität $\left(\mathrm{H}_{3}\right)$}

Der Moderationseffekt der Freundschaftsqualität in der Beziehung zwischen erziehungsbezogener elterlicher Depressivität und kindlicher Selbstwirksamkeitserwartung war positiv, klein bis mittelgroß und signifikant $(\beta=.21, p<.05)$. Die Korrelation zwischen Freundschaftsqualität und erziehungsbezogener elterlicher Depressivität lag bei .00 und war entsprechend nicht signifikant. 


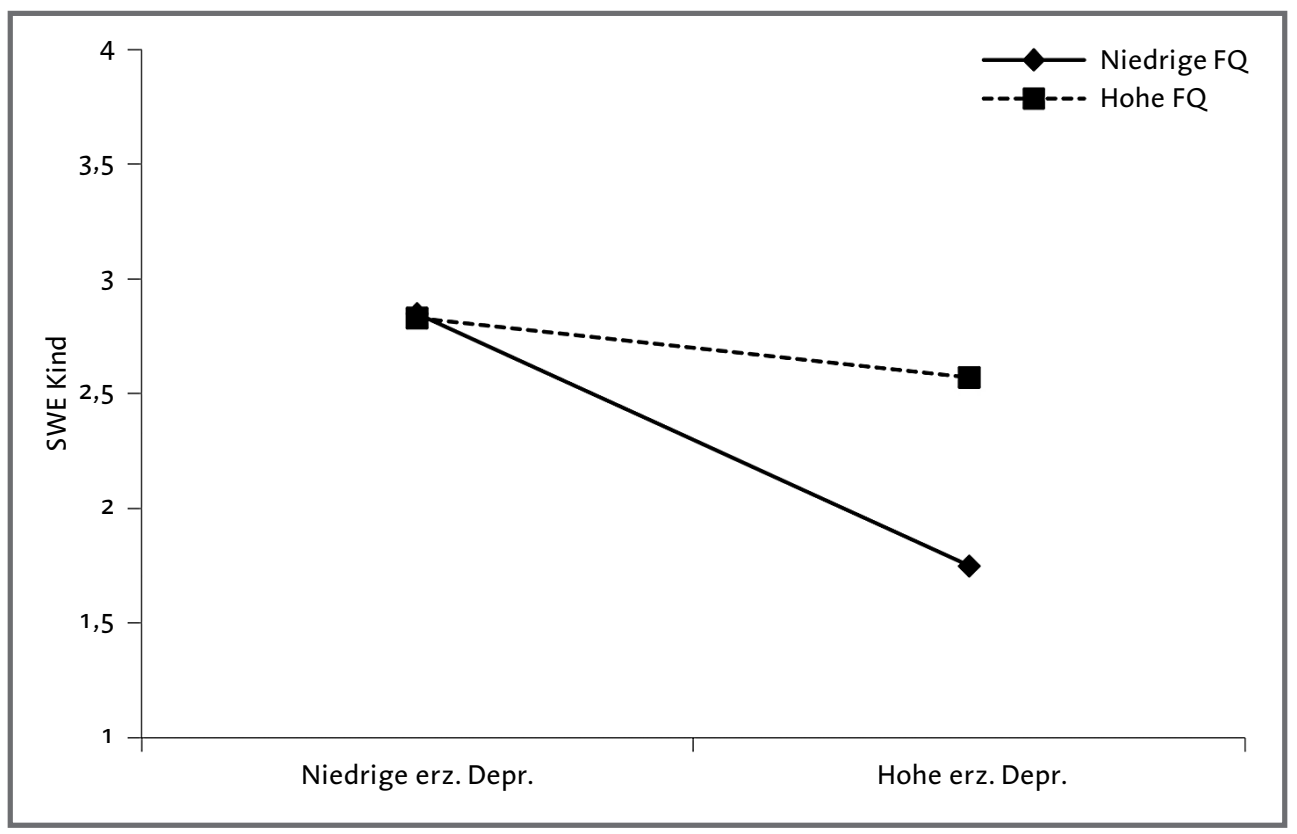

Abb. 2: Zusammenhang zwischen erziehungsbezogener elterlicher Depressivität (erz. Depr.) und kindlicher Selbstwirksamkeitserwartung (SWE Kind) bei hoher und niedriger Freundschaftsqualität (FQ).

In Abbildung 2 wird der Moderationseffekt grafisch dargestellt. Dabei ist ersichtlich, dass sich je nach Zustand und Ausprägung der Freundschaftsqualität der Einfluss der erziehungsbezogenen elterlichen Depressivität auf die kindliche Selbstwirksamkeitserwartung verändert.

\section{Kovariaten - Geschlecht und Alter}

Mädchen wiesen höhere Werte in der Freundschaftsqualität zum besten Freund beziehungsweise zur besten Freundin auf als Jungen $(\mathrm{r}=-.23$, $p<.05)$. Hingegen zeigte sich kein signifikanter Zusammenhang zwischen dem Alter der Kinder und weiteren Variablen.

\section{Diskussion}

Ziel der vorliegenden Studie war es, den Zusammenhang zwischen (1) erziehungsbezogener Depressivität der Eltern und kindlicher Selbstwirksamkeitserwartung zu untersuchen. Dabei wurdegeprüft, inwiefern dieFreundschaftsquali- tät als (2) promotiver und (3) protektiver Faktor für die kindliche Selbstwirksamkeitserwartung identifiziert werden kann.

\section{Haupteffekt der erziehungs- bezogenen elterlichen Depressivität}

Die Ergebnisse der Studie zeigten, dass eine höhere erziehungsbezogene elterliche Depressivität mit weniger Selbstwirksamkeitserwartung der Kinder einhergeht, womit Hypothese 1 bestätigt wurde.

Das Ergebnis steht in Einklang mit den Beobachtungen von Hohm et al. (2017) und Semmelhack (2011), welche bei belastenden Interaktionen zwischen Eltern und Kind sowie bei einer diagnostizierten elterlichen Depression ebenso einen stabil negativen Einfluss auf die kindliche Selbstwirksamkeitserwartung fanden. Der negative Effekt lässt vermuten, dass alle vier Quellen der Selbstwirksamkeitserwartung nach Bandura (1997) durch eine erziehungsbezogene elterliche Depressivität beeinträchtigt werden: 
(1) Direkt erlebte Handlungserfolge und Misserfolge. Eltern mit einer erziehungsbezogenen Depressivität schenken ihren Kindern weniger Vertrauen, sind weniger präsent und räumen den Kindern weniger Raum für Eigeninitiative und Autonomie ein, was die Wahrscheinlichkeit von Handlungsergebnissen der Kinder aufgrund eigener Erfolge sowie das Vertrauen in die eigenen Fähigkeiten hemmt (Lenz \& Kuhn, 2011). Elterliche Selbstzweifel in der Erziehung können sich in Form elterlicher Gehemmtheit und Unsicherheit gegenüber dem Kind zeigen (Tröster, 2011). Das Kind schätzt dadurch sein eigenes Verhalten und Handeln als kritischer ein und führt Handlungserfolge weniger auf eigene Fähigkeiten, sondern auf globale, stabile Ursachen zurück (Schwarzer \& Jerusalem, 2002). (2) Stellvertretende Erfahrungen durch Beobachtung von Verhaltensmodellen. Auch wenn angenommen wird, dass Gleichaltrige als Verhaltensmodelle den größten Einfluss auf die kindliche Selbstwirksamkeitserwartung haben (Schwarzer \& Jerusalem, 2002), wird Eltern als primären Bezugspersonen ein großer Einfluss auf das Verhalten und Erleben der Kinder zugeschrieben (Bowlby, 1969). Bowlby (1969) geht in seiner Bindungstheorie davon aus, dass Kinder interne Arbeitsmodelle in Abhängigkeit von frühen Bindungserfahrungen entwickeln. Diese Arbeitsmodelle beinhalten Repräsentationen des Selbst und beeinflussen die Art und Weise, wie Kinder sich selbst wahrnehmen und erfahren. Ist wenig elterliche Feinfühligkeit vorhanden, wird der Aufbau von Arbeitsmodellen gehemmt, was zu einer niedrigeren Selbstwirksamkeitserwartung führen kann. (3) Verbal ausgedrückte Überzeugungen und Ermutigungen. Die Interaktionsqualität bei Eltern mit einer erziehungsbezogenen Depressivität fällt eher niedrig aus (Weinberg \& Tronick, 1998; Weissman, Paykel \& Klerman, 1972). Augenscheinlich ist dabei, dass Eltern mit einer erziehungsbezogenen Depressivität ihre Kinder seltener ermutigen und in ihren Handlungen bestärken, was die Entwicklung der kindlichen Selbstwirksamkeitserwartung beeinträchtigen kann (Hohm et al., 2017). (4) Wahrnehmungen eigener Gefühlserregung. Eltern mit einer erziehungsbezogenen Depressivität helfen ihren Kindern mangels Feinfühligkeit nur bedingt, ihre Gefühle einzuordnen (Lenz \& Kuhn, 2011; World Health Organization, 2004). Sind Ängste oder Wutgefühle bei Kindern vorhanden, können diese nach Schwarzer und Jerusalem (2002) die Beurteilung der Bewältigungskompetenz und somit der erlebten Selbstwirksamkeit stark einschränken.

\section{Promotiver Effekt der Freundschaftsqualität}

In Übereinstimmung mit den Ergebnissen von Bukowski et al. (2018) und McCauley et al. (2019) zeigten die Ergebnisse der vorliegenden Studie, dass Kinder, welche über eine hohe Freundschaftsqualität zu ihrem besten Freund beziehungsweise ihrer besten Freundin berichten, über eine höhere Selbstwirksamkeitserwartung verfügen (Hypothese 2). Eine hohe Freundschaftsqualität fördert das Vertrauen und die Selbstsicherheit, Herausforderungen standhalten zu können. Durch qualitativ hochwertige Freundschaften entwickeln Kinder sozial-emotionale Kompetenzen und Strategien: Sie lernen, wie sie Emotionen besser ausdrücken und mit verschiedenen Belastungen umgehen können (Bagwell \& Bukowski, 2018; Uchino, Uno \& Holt-Lunstad, 1999). Zudem fühlen sich Kinder mit besten Freunden beziehungsweise besten Freundinnen wertgeschätzt und unterstützt (Bagwell \& Bukowski, 2018; Hoza et al., 2000; Rubin \& Bowker, 2018), was ihre Selbstwirksamkeitserwartung positiv beeinflusst.

\section{Protektiver Effekt der Freundschaftsqualität}

Vorliegend wurde darüber hinaus ein protektiver Effekt der Freundschaftsqualität in der Beziehung zwischen hoher erziehungsbezogener elterlicher Depressivität und kindlicher Selbstwirksamkeitserwartung bestätigt. Das Ergebnis steht in Einklang mit Bagwell und Schmidt (2011), welche mit Verweis auf Stocker (1994) darauf hinwiesen, dass negative familiale Bezie- 
hungserfahrungen durch Freundschaftsbeziehungen hoher Qualität kompensiert werden können. In der vorliegenden Studie war die Korrelation zwischen der Freundschaftsqualität und der erziehungsbezogenen elterlichen Depressivität nicht signifikant. Dies kann als klares Indiz für den Moderationseffekt der Freundschaftsqualität gewertet werden, da die Freundschaftsqualität nicht in Zusammenhang mit der erziehungsbezogenen elterlichen Depressivität steht. Der Moderationseffekt zeigte sich erst bei einer besonders ausgeprägten Form, d. h. einer hohen erziehungsbezogenen elterlichen Depressivität, was darauf hindeutet, dass bei einer niedrigen erziehungsbezogenen elterlichen Depressivität der abpuffernde Einfluss der Freundschaftsqualität auf die Selbstwirksamkeit nicht relevant erscheint. Dies könnte sich dadurch erklären lassen, dass eine niedrige erziehungsbezogene elterliche Depressivität bereits mit einer hohen Selbstwirksamkeitserwartung der Kinder einhergeht und eine hohe Freundschaftsqualität zum besten Freund/zur besten Freundin somit nicht zu einer noch höheren Selbstwirksamkeitserwartung beitragen kann.

Mit Blick auf die vier Quellen der Selbstwirksamkeitserwartung nach Bandura (1997) können beste Freunde hoher Qualität (1) zu konkreten positiven Handlungsergebnissen beitragen (Bukowski et al., 2018): Freunde und Freundinnen haben Ideen im Umgang mit Herausforderungen und können Ratschläge erteilen, was zu eigenen Erfolgen führt. (2) Beste Freunde und Freundinnen sind zumeist in Alter, Geschlecht und weiteren Attributen ähnliche Personen. Aus diesem Grund erzielen sie als Verhaltensmodelle eine besonders gute Wirkung (Schwarzer \& Jerusalem, 2002). Beste Freunde beziehungsweise beste Freundinnen helfen, andere Werte, Haltungen und Überzeugungen kennenzulernen und zu adaptieren, was neue direkte und stellvertretende Erfahrungen ermöglicht (Selman, Levitt \& Schultz, 1997). (3) Beste Freunde beziehungsweise beste Freundinnen schenken Vertrauen und Anerkennung, stehen zur Seite und geben Sicherheit (Schneider, 2016).

(4) In einer besten Freundschaft werden Ge- fühlserregungen ausgetauscht und reguliert (Bagwell \& Bukowski, 2018; Bagwell \& Schmidt, 2011; Hartup, 1989; Sullivan, 1953). Dadurch helfen beste Freunde beziehungsweise beste Freundinnen Kindern, die negativen Auswirkungen mangelnder Feinfühligkeit von Eltern abzupuffern.

\section{Einfluss des Geschlechts}

Während das Alter keinen Einfluss auf die Freundschaftsqualität zeigte, berichten Mädchen von einer höheren Freundschaftsqualität zu ihrem besten Freund beziehungsweise ihrer besten Freundin. Das Ergebnis lässt vermuten, dass der Moderations- beziehungsweise Schutzeffekt bei Mädchen und Jungen unterschiedlich ausfällt, was allerdings vorliegend in Einklang mit Bagwell und Schmidt (2011) nicht bestätigt werden konnte. Die entsprechende Analyse ist in der vorliegenden Studie nicht dargestellt.

\section{Stärken und Grenzen}

Die vorliegende Studie untersuchte erstmals den Einfluss erziehungsbezogener elterlicher Depressivität auf die kindliche Selbstwirksamkeitserwartung. Im Gegensatz zu bisherigen Studien wurde die kindliche Selbstwirksamkeitserwartung als Ergebnisvariable und nicht als Schutzfaktor bei mütterlicher Depression (z. B. Goodman, 2007) einbezogen.

Auch wurde erstmals in diesem Zusammenhang die Rolle der Freundschaftsqualität zum besten Freund beziehungsweise zur besten Freundin als promotiv und protektiv geprüft.

Die Studienergebnisse basieren auf einer querschnittlichen Analyse. Wünschenswert sind an dieser Stelle anknüpfende längsschnittliche Untersuchungen, da die Wirkung von Schutzfaktoren zeitlich instabil sein und sich im Verlauf der kindlichen Entwicklung in ihrer Stärke verändern kann (Bliesener, 2018). Präzisierend muss für die vorliegende Studie festgehalten werden, dass die Einschätzungen der kindlichen Selbstwirksamkeitserwartung durch die Eltern vorgenommen wurden. Es ist denkbar, 
dass Eltern mit einer hohen erziehungsbezogenen Depressivität auch ihre Kinder als weniger selbstwirksam erleben (Webster-Stratton \& Hammond, 1988) und somit die Selbstwirksamkeitserwartung der Kinder eher als elterliches Zutrauen in die Selbstwirksamkeit der Kinder umschrieben werden kann.

Als Einschränkung lässt sich für die Studie anmerken, dass weder untersucht wurde, ob die beste Freundschaft auf Gegenseitigkeit beruht, noch ob der beste Freund beziehungsweise die beste Freundin real oder imaginär sind (z. B. Gleason, 2017; Taylor, Hulette \& Dishion, 2010). Allerdings scheint nach Bagwell und Schmidt (2011) die subjektiv eingeschätzte Freundschaftsqualität, ähnlich wie die selbst wahrgenommene soziale Unterstützung (Eagle, Hybels \& Proeschold-Bell, 2019; Uchino, 2009), als Schutzfaktor wichtiger zu sein als die übereinstimmende, real vorhandene objektive Freundschaftsqualität.

\section{Praktische Implikationen}

Die Resilienzforschung verweist darauf, risikoerhöhende Bedingungen zu reduzieren und vorhandene Ressourcen zu stärken (Masten, 2015; Masten \& Barnes, 2018; Petermann, 2003). Daraus ergeben sich folgende zwei Implikationen: (1) Eltern mit einer hohen erziehungsbezogenen Depressivität in ihrer Reflexionsfähigkeit zu unterstützen, inwiefern ihr Handeln die Selbstwirksamkeitsentwicklung ihres Kindes beeinflusst, sowie die Ursachen ihrer hohen erziehungsbezogenen Depressivität zu ermitteln und zu reduzieren. (2) Kinder in ihren Peerkontakten zu stärken, damit sie positive Beziehungen und Freundschaften aufbauen können. Schulen und Kindertageseinrichtungen können hier einen wichtigen Beitrag leisten.

\section{Danksagung}

Die Autoren bedanken sich bei allen Kindern und Eltern für die Teilnahme an der Studie. Die Studie wurde vom Schweizerischen Nationalfonds (Nr. 100019_166003) und von der Jacobs Foundation finanziell unterstützt.

\section{Literatur}

Abidin, R. R. (1995). Parenting Stress Index. Professional manual (3rd edition.). Odessa, FL: Assessment Resources.

Bagwell, C. L. \& Bukowski, W.M. (2018). Friendship in childhood and adolescence: Features, effects, and processes. In W. M. Bukowksi, B. Laursen \& K. H. Rubin (Eds.), Handbook of peer interactions, relationships, and groups, 2nd ed (p. 371-390). New York, NY, US: The Guilford Press.

Bagwell, C. L. \& Schmidt, M.E. (2011). Friendships in Childhood and Adolescence. New York, NY, US: Guilford Press.

Bakalım, O. \& Karçkay, A.T. (2016). Friendship Quality and Psychological Well-Being: The Mediating Role of Perceived Social Support. International Online Journal of Educational Science, 8(4), 1-9. https://doi.org/10. 15345/iojes.2016.04.001

Bandura, A. (1997). Self-efficacy: The exercise of control. New York: Freeman.

Bandura, A., Pastorelli, C., Barbaranelli, C. \& Caprara, G.V. (1999). Self-efficacy pathways to childhood depression. Journal of Personality and Social Psychology, 76, $258-$ 269. https://doi.org/10.1037/0022-3514.76.2.258

Belsky, J. (1984). The Determinants of Parenting: A Process Model. Child Development, 55(1), 83-96. https://doi. org/10.2307/1129836

Berndt, T. J. (2002). Friendship Quality and Social Development. Current Directions in Psychological Science, 11 (1), 7-10. https://doi.org/10.1111/1467-8721.00157

Bliesener, T. (2018). Resilienz. Schutzfaktoren für delinquentes Handeln. In: D. Hermann \& A. Pöge (Hrgs.): Kriminalsoziologie. Handbuch für Wissenschaft und Praxis (S. 263-276). Baden-Baden: Nomos.

Bloomfield, L. \& Kendall, S. (2012). Parenting self-efficacy, parenting stress and child behaviour before and after a parenting programme. Primary Health Care Research \& Development, 13(4), 364-372. https://doi.org/10.10 $17 / S 1463423612000060$

Bowlby, J. (1969). Attachment and Loss: Attachment. New York: Basic Books.

Bukowski, W. M., Laursen, B. P. \& Rubin, K. H. (Hrsg.). (2018). Handbook of peer interactions, relationships, and groups (Second Edition.). New York: The Guilford Press.

Burke, T., Sticca, F. \& Perren, S. (2017). Everything's gonna be alright! The longitudinal interplay among social support, peer victimization, and depressive symptoms. Journal of Youth and Adolescence, 46 (9), 1999-2014. https://doi.org/10.1007/s10964-017-0653-0

Caprara, G. V., Barbaranelli, C., Pastorelli, C. \& Cervone, D. (2004). The contribution of self-efficacy beliefs to psychosocial outcomes in adolescence: predicting beyond global dispositional tendencies. Personality and Individual Differences, 37(4), 751-763. https://doi.org/ 10.1016/j.paid.2003.11.003

Cuadros, O. \& Berger, C. (2016). The Protective Role of Friendship Quality on the Wellbeing of Adolescents Victimized by Peers. Journal of Youth and Adolescence, 45(9), 1877-1888. https://doi.org/10.1007/s10964016-0504-4

Cummings, E. M. \& Davies, P.T. (1994). Maternal depression and child development. Journal of child psychology and psychiatry, 35, 73-122. https://doi.org/10.1111/ j.1469-7610.1994.tb01133.x 
Demir, M., Özdemir, M. \& Weitekamp, L.A. (2007). Looking to happy tomorrows with friends: Best and close friendships as they predict happiness. Journal of Happiness Studies, 8(2), 243-271. https://doi.org/10.1007/ s10902-006-9025-2

Dutra-Thomé, L., DeSousa, D. \& Koller, S. H. (2018). Promotive and risk factors for positive youth development among emerging adults in brazil. Child \& Youth Care Forum, 38, 171 - 185. https://doi.org/10.1007/s105 66-018-9475-9

Eagle, D. E., Hybels, C. F. \& Proeschold-Bell, R. J. (2019). Perceived social support, received social support, and depression among clergy. Journal of Social and Personal Relationships, 36(7), 2055-2073. https://doi.org/10. $1177 / 0265407518776134$

Ebner, K., Schulte, E.-M., Soucek, R. \& Kauffeld, S. (2018). Coaching as stress-management intervention: The mediating role of self-efficacy in a framework of self-management and coping. International Journal of Stress Management, 25(3), 209-233. https://doi.org/ $10.1037 /$ str0000058

Furman, W. \& Buhrmester, D. (1985). Children's perceptions of the personal relationships in their socia networks. Developmental Psychology, 21, 1016-1024. https://doi.org/10.1037/0012-1649.21.6.1016

Gaertner, A. E., Fite, P. J. \& Colder, C. R. (2010). Parenting and friendship quality as predictors of internalizing and externalizing symptoms in early adolescence. Journal of Child and Family Studies, 19(1), 101-108. https:// doi.org/10.1007/s10826-009-9289-3

Gelfand, D.M. \& Teti, D. M. (1990). The effects of maternal depression on children. Clinical Psychology Review, 10(3), 329-353. https://doi.org/10.1016/02727358(90)90065-I

Gleason, T.R. (2017). The psychological significance of play with imaginary companions in early childhood. Learning \& Behavior, 45(4), 432-440. https://doi. org/10.3758/s13420-017-0284-z

Goodman S. H. (2007). Depression in mothers. Annual review of clinical psychology, 3, 107-135. https://doi.org/ 10.1146/annurev.clinpsy.3.022806.091401

Graber, R., Turner, R. \& Madill, A. (2016). Best friend and better coping: Facilitating psychological resilience through boys' and girls' closest friendships. British Journal of Psychology, 107(2), 338-358. https://doi.org/ 10.1111 /bjop. 12135

Hair, J. F. (Hrsg.). (2014). Multivariate data analysis (Pearson custom library) (7. ed., Pearson new internat. ed.). Harlow: Pearson.

Hammen, C. (1997). Children of Depressed Parents (Issues in Clinical Child Psychology). In S. A. Wolchik \& I. N Sandler (Eds.), Handbook of Children's Coping: Linking Theory and Intervention (p. $131-157)$. Boston, MA Springer US. https://doi.org/10.1007/978-1-4757-26 77-0 5

Hartup, W. W. (1989). Social relationships and their developmental significance. American Psychologist, 44, 120 126. https://doi.org/10.1037/0003-066X.44.2.120

Havighurst, R. J. (1976). Developmental tasks and education (3rd ed.). New York: McKay.

Hohm, E., Laucht, M., Zohsel, K., Schmidt, M. H., Esser, G., Brandeis, D. \& Banaschewski, T. (2017). Resilienz und Ressourcen im Verlauf der Entwicklung: Von der frühen Kindheit bis zum Erwachsenenalter. Kindheit und Entwicklung, 26, 230-239. https://doi.org/10.10 26/0942-5403/a000236
Hoza, B., Bukowski, W. M. \& Beery, S. (2000). Assessing Peer Network and Dyadic Loneliness. Journal of Clinical Child Psychology, 29(1), 119-128. https://doi.org/ $10.1207 / S 15374424 j \mathrm{ccp} 2901 \_12$

Hu, L. \& Bentler, P. M. (1999). Cutoff criteria for fit indexes in covariance structure analysis: Conventional criteria versus new alternatives. Structural Equation Modeling: A Multidisciplinary Journal, 6(1), 1-55. https:// doi.org/10.1080/10705519909540118

Hulland, J. (1999). Use of Partial Least Squares (PLS) in Strategic Management Research: A Review of Four Recent Studies. Strategic Management Journal, 20, 195-204. https://doi.org/10.1002/(SICI)1097-0266(199902) 20:2<195::AID-SMJ13>3.0.CO;2-7

Jennings, K. D. \& Abrew, A. J. (2004). Self-efficacy in 18month-old toddlers of depressed and nondepressed mothers. Journal of Applied Developmental Psychology, 25(2), 133-147. https://doi.org/10.1016/j.appdev.2004.02 001

Jerusalem, M. \& Schwarzer, R. (1999). Skalen zur Erfassung von Lehrer-und Schülermerkmalen. Dokumentation der psychometrischen Verfahren im Rahmen der Wissenschaftlichen Begleitung des Modellversuchs Selbstwirksame Schulen. Berlin: Freie Universität Berlin.

Kline, R. B. (2016). Principles and practice of structural equation modeling, 4th ed. New York, NY, US: Guilford Press.

Kotov, R., Krueger, R. F., Watson, D., Achenbach, T. M. Althoff, R. R., Bagby, R. M.,...Zimmermann, M. (2017). The Hierarchical Taxonomy of Psychopathology (HiTOP): a dimensional alternative to traditional nosologies. Journal of Abnormal Psychology, 126(4), 454-477. https://doi.org/10.1037/abn0000258

Kötter, C., Stemmler, M., Bühler, A. \& Lösel, F. (2010). Mütterliche Depressivität, Erziehung und kindliche Erlebens- und Verhaltensprobleme. Kindheit und Entwicklung, 19(2), 109-118. https://doi.org/10.1026/ 0942-5403/a000006

Lansford, J. E., Criss, M. M., Pettit, G. S., Dodge, K. A. \& Bates, J. E. (2003). Friendship Quality, Peer Group Affiliation, and Peer Antisocial Behavior as Moderators of the Link Between Negative Parenting and Adolescent Externalizing Behavior. Journal of research on adolescence: the official journal of the Society for Research on Adolescence, 13(2), 161-184.

Laucht, M., Esser, G. \& Schmidt, M. H. (2000). Entwicklung von Risikokindern im Schulalter: Die langfristigen Folgen frühkindlicher Belastungen. Zeitschrift für Entwicklungspsychologie und pädagogische Psychologie, 32(2), 59-69. https://doi.org/10.1026//0049-8637. 32.2 .59

Lenz, A. (2008). Interventionen bei Kindern psychisch kranker Eltern - Grundlagen, Diagnostik und therapeutische Maßnahmen. Göttingen: Hogrefe Verlag.

Lenz, A. (2014). Kinder psychisch kranker Eltern. Göttingen Hogrefe Verlag.

Lenz, A. \& Kuhn, J. (2011). Was stärkt Kinder psychisch kranker Eltern und fördert ihre Entwicklung? Überblick über die Ergebnisse der Resilienz- und Copingforschung. In S. Wiegand-Grefe, F. Mattejat \& A. Lenz (Hrsg.), Kinder mit psychisch kranken Eltern. Klinik und Forschung (S. 269-298). Göttingen: Vandenhoeck \& Ruprecht.

Little, T. D. (2013). Longitudinal Structural Equation Modeling. New York: Guilford Press.

Masten, A. S. (2015). Ordinary magic: Resilience in development. The Guilford Press. 
Masten, A. S. \& Barnes, A. J. (2018). Resilience in children: Developmental perspectives. Children, 5(7), 1-16. https://doi.org/10.3390/children5070098

Masten, A. S., Best, K. M. \& Garmezy, N. (1990). Resilience and development: Contributions from the study of children who overcome adversity. Development and Psychopathology, 2, 425-444. https://doi.org/10.1017/ S0954579400005812

McCauley, D. M., Weymouth, B. B., Feinberg, M. E. \& Fosco, G.M. (2019). Evaluating school and peer protective factors in the effects of interparental conflict on adolescent threat appraisals and self-efficacy. Journal of Adolescence, 71, 28-37. https://doi.org/10.1016/j.adole scence.2018.12.005

Muthén, L. K. \& Muthén, B. O. (1998-2017). Mplus User's Guide. Eighth Edition. Los Angeles, CA: Muthén \& Muthén.

Nangle, D. W., Erdley, C. A., Newman, J. E., Mason, C. A. \& Carpenter, E. M. (2003). Popularity, friendship quantity, and friendship quality: Interactive influences on children's loneliness and depression. Journal of Clinical Child and Adolescent Psychology, 32 (4), 546-555. https://doi.org/10.1207/S15374424JCCP3204_7

Oldenburg, C. M. \& Kems, K. A. (1997). Associations between Peer Relationships and Depressive Symptoms: Testing Moderator Effects of Gender and Age. The Journal of Early Adolescence, 17(3), 319-337. https:// doi.org/10.1177/0272431697017003004

Östberg, M. (1998). Parental stress, psychosocial problems and responsiveness in help-seeking parents with small (2-45 months old) children. Acta Paediatrica, 87(1) 69-76. https://doi.org/10.1111/j.1651-2227.1998. tb01389.x

Parker, J. G. \& Asher, S. R. (1993). Friendship and friendship quality in middle childhood: Links with peer group acceptance and feelings of loneliness and social dissatisfaction. Developmental Psychology, 29, 611-621. https://doi.org/10.1037/0012-1649.29.4.611

Petermann, F. (2003). Prävention und Verhaltensstörungen - Einführung in den Themenschwerpunkt. Kindheit und Entwicklung, 12(2), 65-70.

Rodebaugh, T. L., Lim, M. H., Shumaker, E. A., Levinson, C.A. \& Thompson, T. (2015). Social Anxiety and Friendship Quality over Time. Cognitive Behaviour Therapy, 44(6), 502-511. https://doi.org/10.1080/1 6506073.2015.1062043

Rubin, K.H. \& Bowker, J.C. (2018). Friendships. In M. Bornstein, M. E. Arterberry, K. L. Fingerman \& J. E. Lansford (Eds.), The SAGE Encyclopedia of Lifespan Human Development. California: SAGE Publications. https://doi.org/10.4135/9781506307633. n339

Rutter, M. (1990). Psychosocial Resilience and Protective Mechanisms. In J. E. Rolf, A. S. Masten, D. Cichetti \& K. H. Nuechterlein (Hrsg.), Risk And Protective Factors in the Development of Psychopathology (S. 181-214). New York: Cambridge University Press.

Sanderson, J.A. \& Siegal, M. (1995). Loneliness and stable friendship in rejected and nonrejected preschoolers Journal of Applied Developmental Psychology, 16(4), 555567. https://doi.org/10.1016/0193-3973(95)90004-7

Schaunig, I., Willinger, U., Diendorfer-Radner, G., Hager, V., Jörgl, G., Sirsch, U. \& Sams, J. (2004). Parenting Stress Index: Einsatz bei Müttern sprachentwicklungsgestörter Kinder. Praxis der Kinderpsychologie und Kinderpsychiatrie, 53(6), 395-405.
Scheithauer, H. \& Petermann, F. (1999). Zur Wirkungsweise von Risiko- und Schutzfaktoren in der Entwicklung von Kindern und Jugendlichen. Kindheit und Entwicklung, 8, 3-14. https://doi.org/10.1026//0942-5403. 8.1.3

Schmidt, M. E. \& Bagwell, C. L. (2007). The Protective Role of Friendships in Overtly and Relationally Victimized Boys and Girls. Merrill-Palmer Quarterly, 53 , 439-460. https://doi.org/10.1353/mpq.2007.0021

Schmitt, M. \& Altstötter-Gleich, C. (2010). Differentielle Psychologie und Persönlichkeitspsychologie kompakt-Mit Online-Materialien. Weinheim: Julius Beltz GmbH \& Co. KG.

Schneider, B.H. (2016). Childhood friendships and peer relations: friends and enemies (Second Edition.). London; New York: Routledge, Taylor \& Francis Group. https://doi.org/10.4324/9781315727042

Schwarzer, R. \& Jerusalem, M. (2002). Das Konzept der Selbstwirksamkeit. Zeitschrift für Pädagogik (Beiheft; 44), $28-53$.

Selman, R., Levitt, M. Z. \& Schultz, L. H. (1997). The Friendship Framework: Tools for the Assessment of Psychosocial Development. In R. Selman (Ed.), Fostering Friendship. Pair Therapy for Treatment and Prevention (p. 31-52). New York: Routledge. https://doi.org/10. 4324/9781351327442-4

Semmelhack, C. (2011). Ein „Forum" für Kinder psychisch kranker Eltern: Die Lebenswelt der Kinder und ein Ansatz zur Ergänzung sozialpädagogischer Angebote mittels neuer Medien. Hamburg: Kovac.

Simoni, H., Herren, J., Kappeler, S. \& Licht, B. (2015). Frühe soziale Kompetenz unter Kindern. In T. Malti \& S. Perren (Hrsg.), Entwicklung und Förderung sozialer Kompetenzen in Kindheit und Adoleszenz (2. überarbeitete Auflage., S. 15-35). Stuttgart: Kohlhammer.

Stocker, C. M. (1994). Children's perceptions of relationships with siblings, friends, and mothers: Compensatory processes and links with adjustment. Child Psychology \& Psychiatry \& Allied Disciplines, 35 (8), 1447-1459. https://doi.org/10.1111/j.1469-7610.1994.tb01286.x

Sullivan, H. S. (1953). The interpersonal theory of psychiatry. New York: Norton.

Taylor, M., Hulette, A. C. \& Dishion, T. J. (2010). Longitudinal outcomes of young high-risk adolescents with imaginary companions. Developmental Psychology, 46 (6), 1632-1636. https://doi.org/10.1037/a0019815

Traub, A. (2006). Kontinuität und Kompensation. Die Bedeutung von Familie und Gleichaltrigen (Peers) für Persönlichkeit und Problemverhalten in der mittleren Kindheit. Diskurs Kindheits-und Jugendforschung, 1 (2), $197-216$.

Tröster, H. (2011). Eltern-Belastungs-Inventar (EBI), Deutsche Version des Parenting Stress Index (PSI) von R. R. Abidin. Göttingen: Hogrefe.

Uchino, B. N. (2009). Understanding the links between social support and physical health: a life-span perspective with emphasis on the separability of perceived and received support. Perspectives on Psychological Science, 4(3), 236-255. https://doi.org/10.1111/j.1745-6924. 2009.01122.x

Uchino, B. N., Uno, D. \& Holt-Lunstad, J. (1999). Social support, physiological processes, and health. Current Directions in Psychological Science, 8(5), 145-148. https://doi.org/10.1111/1467-8721.00034 


\section{Olivia Gasser-Haas, Fabio Sticca, Corina Wustmann Seiler}

Venkataraman, M. \& Ackerson, B. J. (2008). Parenting Among Mothers With Bipolar Disorder: Strengths, Challenges, and Service Needs. Journal of Family Social Work, 11 (4), 389-408. Routledge. https://doi.org/ $10.1080 / 10522150802441825$

Waldrip, A. M., Malcolm, K. T. \& Jensen-Campbell, L. A. (2008). With a Little Help from Your Friends: The Importance of High-quality Friendships on Early Adolescent Adjustment. Social Development, 17(4), 832-852. https://doi.org/10.1111/j.1467-9507.2008.00476.x

Webster-Stratton, C. \& Hammond, M. (1988). Maternal depression and its relationship to life stress, perceptions of child behavior problems, parenting behaviors, and child conduct problems. Journal of Abnormal Child Psychology, 16(3), 299-315. https://doi.org/10.1007/ BF00913802

Weinberg, M. K. \& Tronick, E.Z. (1998). Emotional characteristics of infants associated with maternal depression and anxiety. Pediatrics, 102 (5 Suppl E), 1298-1304.

Weissman, M., Paykel, E. \& Klerman, G. (1972). The depressed woman as a mother. Social Psychiatry, 7, 98 108. https://doi.org/10.1007/BF00583985
World Health Organization. (2004). The importance of caregiver-child interactions for the survival and healthy development of young children: a review. Geneva: World Health Organization.

Wustmann Seiler, C. W., Müller, E. \& Simoni, H. (2017) The Protective Role of Childcare Quality for Behavioral Adjustment in 3- to 5-Year-Old Children. Zeitschrift für Entwicklungspsychologie und Pädagogische Psychologie, 49, 1-10. https://doi.org/10.1026/0049-8637/ a000162

Wustmann Seiler, C. (2020). Resilienz: Widerstandsfähigkeit von Kindern in Tageseinrichtungen fördern. Beiträge zur Bildungsqualität (8. Auflage.). Berlin: Cornelsen Scriptor.

\section{Olivia Gasser-Haas}

Marie Meierhofer Institut für das Kind

Pfingstweidstr. 16

$\mathrm{CH}-8005$ Zürich

Tel. (044) 2055220

E-Mail: gasser@mmi.ch 